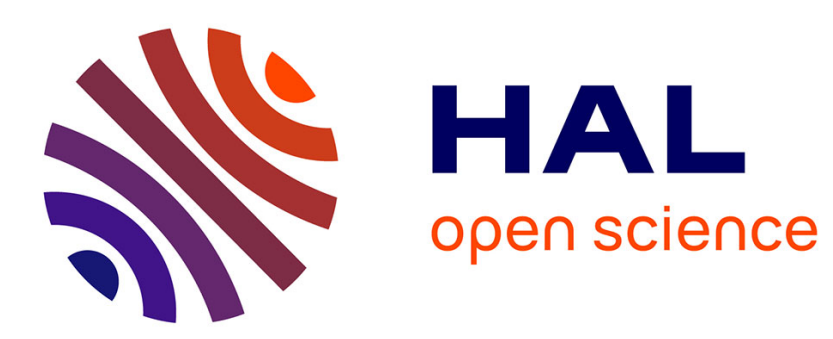

\title{
Internal variables and phenomenological models for metals plasticity \\ F. Sidoroff
}

\section{To cite this version:}

F. Sidoroff. Internal variables and phenomenological models for metals plasticity. Revue de Physique Appliquée, 1988, 23 (4), pp.649-659. 10.1051/rphysap:01988002304064900 . jpa-00245813

\section{HAL Id: jpa-00245813 https://hal.science/jpa-00245813}

Submitted on 1 Jan 1988

HAL is a multi-disciplinary open access archive for the deposit and dissemination of scientific research documents, whether they are published or not. The documents may come from teaching and research institutions in France or abroad, or from public or private research centers.
L'archive ouverte pluridisciplinaire HAL, est destinée au dépôt et à la diffusion de documents scientifiques de niveau recherche, publiés ou non, émanant des établissements d'enseignement et de recherche français ou étrangers, des laboratoires publics ou privés. 


\title{
Internal variables and phenomenological models for metals plasticity
}

\author{
F. Sidoroff
}

Laboratoire de Mécanique des Solides, Ecole Centrale de Lyon, GRECO, 36 avenue Guy de Collongue, B.P. 163, 69131 Ecully Cedex

(Reçu le 15 juin 198\%, révisé le 4 janvier 1988, accepté le 7 janvier 1988)

\begin{abstract}
RESUME.- L'objectif de cette présentation est de dégager la structure générale des modèles phénoménologiques habituellement utilisés pour la description macroscopique du comportement plastique des métaux. Ceci permettra de discuter l'interaction de ces modèles avec les travaux menés sur l'analyse microstructurale. On se limitera aux modèles avec variables internes, car ceux-ci doivent par principe inclure dès le début une certaine vision de l'état microstructural du matériau. Après avoir présenté le cadre mécanique et thermodynamique de base, on décrira les modèles classiques au travers des variables internes qu'ils introduisent, des forces thermodynamiques associées et des mécanismes microscopiques qu'elles sont censées prendre en compte. On présentera ensuite les approches qui permettent de donner à ces modèles un contenu microstructural plus grand. On établira une distinction entre l'approche micromécanique et l'utilisation phénoménologique d'informations microstructurales. Cette discussion méthodologique sera illustrée de quelques modèles tirés de travaux récents. On concluera par quelques exemples combinant ces deux approches. On discutera également la notion de contrainte interne. En conclusion, on plaidera pour un rapprochement entre la mécanique et la physique des matériaux.
\end{abstract}

ABSTRACT. - This paper aims towards a presentation of the general structure of the phenomenological models presently used for the macroscopic description of metal plasticity and a discussion of their interaction with microstructural investigation. Attention is concentrated on internal variables modelization which, from the very beginning, must include some ideas about the microstructural material state. After a presentation of the basic mechanical and thermodynamical framework, the classical models will be described through the usual internal variables, the associated thermodynamic forces and the microstructural features they are supposed to take into account. Some approaches will then be presented to give a deeper microstructural content to these models. A distinction will be made between micromechanical approaches and qualitative use of microstructural informations. This methodological discussion will be illustrated by some recent works and models, and concluded by some examples showing the necessity to combine these two approaches. The concept of internal stress will also be discussed. As a conclusion the need for an increasing interaction between the mechanics and physics of materials will be advocated.

\section{INTRODUCTION}

A considerable amount of work has been expended, in recent years, about the mechanical and phenomenological modelization of plastic materials and in particular of metals. The development of numerical analysis and finite elements methods, the ever increasing capacity and speed of modern computers on one hand, the practical requirement of a better knowledge and prediction of the behaviour of engineering structures on the other hand, a 11 these factors have created a large demand for physically sound and reasonably accurate constitutive equations. Much progress has been achieved [1], but the behaviour of metals under complex or cyclic loading may be very complicated and many aspects cannot yet be taken into account.

In the same time, similar progress have been made about the microstructural characterization and understanding of the basic mechanisms which are responsible for this behaviour. The conditions for a fruitful interaction between these two approaches - Continuum Mechanics and Physical Metallurgy - are therefore created: sufficient and similar development of each discipline, together with an increasing need for complementarity. The discussion has been opened, a common language has often been found leading to a better understanding and new ideas. 
However the complete association of mechanics with physics towards the formulation of a complete and practically useable constitutive model remains an accident. Examples can be found and some of them will be discussed later, but they are isolated. The future development of this kind of approach requires a clear understanding of the general structure of a constitutive model and some kind of strategy about where and how microstructural informations are required or may be used. The purpose of the present paper is to propose such a general approach to constitutive equations. They will be presented from the mechanical point of view, but attention will be concentrated on methodology rather than on technical aspects which are often important but inessential here.

\section{CONSTITUTIVE EQUATIONS}

\subsection{GENERAL FRAMEWORK}

For structural analysis purpose, the behaviour of a material is described by the constitutive equation which is a relation between the local stress $\sigma$ and strain $\varepsilon$. These are usually taken as two or three dimensional tensors, and their precise definition may be part of the problem, in particular in case of large strain [2], but this is not important here. For the sake of definiteness, we shall consider here that $\sigma$ and $\varepsilon$ respectively denote the usual stress and strain tensors, but the formalism which will be used may be applied to different situations.

In an elastic material, the stress $\sigma(t)$ at time $t$ only depends on the strain $\varepsilon(t)$ at the same time

$$
\sigma(t)=\Sigma(\varepsilon(t))
$$

But, as soon as there is some kind of non elasticity or irreversibility, this is no longer true and the stress $\sigma(t)$ also depends on the past deformation history $\varepsilon(\tau)$ for $\tau<t$. The constitutive function $\Sigma$ in (1) must then be replaced by a constitutive functional

$$
\sigma(t)=\underset{\tau \leq t}{G}\{\varepsilon(\tau)\}
$$

In case of linear viscoelasticity for instance, this leads to the classical Volterra's integral, but more generally a constitutive model may be viewed as a causal hereditary system.

A similar formulation may be used for thermomechanical models which must give the stress $\sigma(t)$ and volumic entropy $\eta(t)$ as depending on the strain and temperature history, $\varepsilon(\tau), \theta(\tau)$. The second law of thermodynamics is then usually formulated as the Clausius-Duhem inequality [3], [1]

$$
-(\dot{\psi}+\eta \dot{\theta})+\sigma \dot{\varepsilon}-q \cdot \operatorname{grad} \theta / \theta \geq 0
$$

where $\psi$ is the volumic free energy and $q$ the heat flux. The constitutive model must then be complemented by a similar constitutive relation for $\psi$ and some kind of Fourier's law relating the heat flux $q$ to the temperature gradient.

\subsection{INTERNAL VARIABLES}

The hereditary formulation (2), which is quite appropriate in viscoelasticity, is of little help in case of plastic or viscoplastic behaviour.
Some attempts towards hereditary plasticity have been made [4], [5], but the internal variables approach is usually preferred because of its clearer physical meaning: the present stressstrain relation depends on the past history through the present value of a set of internal state variables $\boldsymbol{\omega}(t)$. These variables are a macroscopic description of the internal microstructural state of the material which in turn depends on the past history. They are sometimes called hidden variables to emphasize the fact that they cannot be controlled.

The constitutive equation (2) can then be rewritten as

$$
\sigma(t)=\Sigma(\varepsilon(t), \omega(t), \varepsilon(t))
$$

In the general case this will result in a viscous behaviour without instantaneous elasticity. On the contrary, if the strain rate $\dot{\varepsilon}$ does not occur in (4), the behaviour is non viscous and can support instantaneous deformations.

This constitutive equation must be supplemented by an evolution equation for these internal variables

$$
\dot{\omega}=q(\varepsilon(t), \omega(t))
$$

in case of rate dependent (viscoplastic) behaviour, or

$$
\dot{\omega}=\lambda Q(\omega(t), \varepsilon(t)) \quad G(\varepsilon(t), \omega(t))=0
$$

in case of rate independent (plastic) behaviour with an arbitrary plastic multiplier $\lambda$ and a yield function $\mathbb{F}$.

It is clear, from its basic assumptions, that this kind of model will be able to include microstructural information and this will be developed in the following. It must also be noted that this approach is elemental: each elementary mechanism will be represented by one internal variable, and a complete model will result from the combination of the appropriate variables. The usual rheological models are the simplest examples of this combination process. It must also be emphasized that the same material will usually be described by different constitutive models according to the kind of problem investigated and to the loading conditions (temperature, loading rate,...). A complete constitutive model for one material under all possible conditions does not exist.

\subsection{GENERALIZED STANDARD MATERIALS}

Some macroscopic guidelines for the formulation of a specific model can be obtained from general principles and in particular from the dissipation inequality (3) or its mechanical counterpart

$$
-\dot{\psi}+\sigma \dot{\varepsilon} \geq 0
$$

The energy $\psi$ depends on the thermodynamical state and therefore on $\varepsilon(t), \omega(t)$. Substitution of this function $\psi(\varepsilon, \omega)$ in (7) leads to

$$
\begin{aligned}
& \sigma^{\vee} \cdot \dot{\varepsilon}+H . \dot{\omega} \geq 0, \\
& \sigma^{\vee}=\sigma-\partial \psi / \partial \varepsilon, \quad H=-\partial \psi / \partial \omega
\end{aligned}
$$

where $\sigma^{v}$ is a viscous stress and $H$ the thermodynamic force associated to the internal variables $\omega$. 
The generalized standard formulation provides a very convenient framework for the derivation of constitutive models. It results from the application of non-linear irreversible thermodynamics [3], [6] to the dissipation inequality (8). It is based on the introduction of a convex dissipation potential $\Omega(\dot{x})$

$$
\Omega=\Omega(\dot{\varepsilon}, \dot{\omega}) \quad \sigma^{V}=\partial \Omega / \partial \dot{\varepsilon} \quad H=\partial \Omega / \partial \dot{\omega}
$$

in the general viscous case, while $\Omega=\Omega(\dot{\omega})$ and $\sigma^{v}=0$ in the non viscous case. If $\Omega$ is a quadratic function, (9) result in the linear Onsager's relations. If $\Omega$ is an homogeneous function of degree one, a rate independent behaviour is obtained. In the other cases this results in a non linear viscoplastic law.

In the non-viscous case, it is convenient to use instead of $\Omega$ the conjugate potential

$$
\stackrel{*}{\Omega}(H)=\sup _{\dot{\omega}}(H \dot{\omega}-\Omega(\dot{\omega}))
$$

which allows a direct expression of the evolution equation as

(11) $\dot{\omega}=\partial \stackrel{*}{\Omega} / \partial \mathrm{H}$

in the rate dependent case, while the rate independent case introduces a yield function $\mathcal{F}_{(}(\mathrm{H})$ such that

(12) $\dot{\omega}=\lambda \partial F / \partial H$

$G(H) \leq 0$

which obviously is a special case of (6).

This formalism is very convenient because a complete model is entirely defined from the construction and identification of the two functions $\psi(\varepsilon, \omega)$ and $\delta(H)$.

\section{THE CLASSICAL MODELS}

An internal variables model essentially includes three components:

1) A choice of internal variables $\omega$,

2) A constitutive equation (4),

3) An evolution equation (5) or (6).

The classical models are obtained from the combination of elementary models, i.e. of elementary internal variables or mechanisms. We shall now describe the structure of these classical elementary models.

\subsection{PLASTIC DEFORMATIONS}

The first basic idea, in the description of plasticity, is the decomposition of the total strain $\varepsilon$ into an elastic and a plastic part

$$
\varepsilon=\varepsilon^{e}+\varepsilon^{p}
$$

From a phenomenological point of view, the plastic deformation is defined as the residual strain which remains after a local unloading process, and it is the first variable to be included in $\omega$. It is not, however, a state variable, and this decomposition is usually complemented by the assumption that the constitutive equation does not depend on $\varepsilon$ and $\varepsilon^{p}$ but only on the elastic strain $\varepsilon^{e}$. The constitutive equation (4) and evolution equation (5) or $(6)$ then reduce to

$$
\begin{aligned}
& \sigma=\Sigma\left(\varepsilon^{e}, \ldots\right) \\
& \dot{\varepsilon}^{p}=m\left(\varepsilon^{e}, \ldots\right) \text { or } \lambda M\left(\varepsilon^{e}, \ldots\right)
\end{aligned}
$$

It must be noted here that a non-viscous behaviour is assumed (instantaneous reversible elastic behaviour). These equations are often used in an equivalent inverted form

$$
\begin{aligned}
& \varepsilon^{e}=\Lambda(\sigma, \ldots) \\
& \dot{\varepsilon}^{p}=m(\sigma, \ldots) \text { or } \lambda M(\sigma, \ldots)
\end{aligned}
$$

From a thermodynamical point of view, the energy $\psi$ depends on the state variable $\varepsilon^{e}$ and the dissipation inequality (7) reduces to

$$
\left(\sigma-\partial \psi / \partial \varepsilon^{e}\right) \dot{\varepsilon}^{e}+\sigma \dot{\varepsilon}^{p}+\ldots \geq 0
$$

Elastic reversibility requires the vanishing of the first term, resulting in the usual elastic law

(17) $\sigma=\partial \psi / \partial \varepsilon^{e}=\sigma\left(\varepsilon^{e}, \ldots\right)$

while the dissipation is purely plastic with the stress $\sigma$ as thermodynamic force associated to the plastic strain rate $\dot{\varepsilon}^{p}$. Application of the generalized standard evolution equations (11) and (12) then results in the usual standard plastic or viscoplastic flow rule

$$
\text { (18) } \quad \dot{\varepsilon}^{p}=\partial \Omega / \partial \sigma \quad \text { or } \lambda \partial F / \partial \sigma \quad G(\sigma, \ldots) \leq 0
$$

which obviously is a special case of (15).

In fact, the generalized standard formulation has beer postulated as a generalization of (18) to all kinds of internal variables.

From a practical point of view, the identification of a plastic model requires the determination of the elastic response and of the yield condition GF. The latter is usually formulated in terms of a tensile equivalent stress

$$
g(\sigma)=\bar{\sigma}-\sigma_{0} \quad \bar{\sigma}=\bar{\sigma}(\sigma)
$$

Viscoplastic identification requires the determination of $\Omega$ which is also usually postulated as depending on $\bar{\sigma}$. This allows the identification of $\Omega(\sigma)$ from purely tensile tests. In case of plastic incompressibility, the equivalent stress $\bar{\sigma}$ only depends on the stress deviator $\sigma^{D}$ and the von Mises and Hill's forms are the most commonly used

(20) $\bar{\sigma}=\sqrt{2 / 3}\left|\sigma^{D}\right|$ or $\sqrt{\sigma^{D}: H: \sigma^{D}}$

From a physical point of view, finally, $\varepsilon^{e}$ represents the elastic or thermoelastic lattice deformation, while the plastic strain $\varepsilon^{p}$ describes the deformation resulting from the motion of dislocations and other crystal defects. At least in the simplest case, where dislocation gliding is the primary deformation mechanism, the general structure presented above can be viewed as the macroscopic extension of natural single crystal properties [7].

An important special case is the rigid plastic approximation which neglects the elastic deformation $\varepsilon^{e}$. In this case, $\varepsilon=\varepsilon^{p}$, and (15) reduces to 


$$
\begin{aligned}
& \dot{\varepsilon}=m(\sigma, \ldots) \quad \text { or } \lambda M(\sigma, \ldots) \\
& \sigma=\Sigma(\dot{\varepsilon}, \ldots)
\end{aligned}
$$

so that plasticity and viscoplasticity appears as some kind of highly non linear fluid behaviour. In this case, $\varepsilon^{\mathfrak{p}}$ disappears from $\omega$, but a viscous behaviour with dependence on $\dot{\varepsilon}$ must then be considered.

\subsection{ISOTROPIC HARDENING}

When the yield function $g$ or viscoplastic potential $\Omega$ only depends on $\sigma$, a perfect plastic behaviour is obtained. The description of hardening requires the parametrization of their evolution through supplementary hardening variables. The simplest case is the isotropic hardening which assumes an homothetic evolution of the yield surface $F=0$ or of the equipotentials $\Omega=$ Const. in the stress space.

Let us consider first the rate independent plastic case with a yield condition expressed as (19). Isotropic hardening will be obtained by assuming the yield limit $\sigma_{0}$ to be a function of some scalar parameter $p$ which may be for instance the cumulated plastic strain or the plastic work. The generalized standard formulation can here be used without loss in generality. The energy is then taken as

$$
\psi=\psi\left(\varepsilon^{e}, p\right)=\psi^{e}\left(\varepsilon^{e}\right)+\psi^{h}(p)
$$

where no coupling has been assumed between elasticity and hardening. Substitution of this form in the dissipation inequality gives the elastic law (17) and

$$
\text { (23) } \sigma \dot{\varepsilon}^{p}-R \dot{p} \geq 0 \quad R=d \psi^{h} / d p=R(p)
$$

The thermodynamic force $H$ associated to $\omega=\left(\varepsilon^{p}, p\right)$ is $(0,-R)$ and, following (12), the yield function is postulated as $F(H)$. Isotropic hardening will be obtained for

$$
\begin{aligned}
& F(\sigma, R)=\bar{\sigma}(\sigma)-\sigma_{0}-R \leq 0 \\
& \dot{\varepsilon}^{p}=\lambda \partial \bar{\sigma} / \partial \sigma \quad \dot{p}=-\lambda \partial F_{F} / \partial R=\lambda
\end{aligned}
$$

If von Mises' equivalent stress is used, then it is easily shown that $p$ can be identified to von Mises' equivalent strain

$$
\dot{p}=\dot{\bar{\varepsilon}}=\sqrt{2 / 3}\left|\dot{\varepsilon}^{p}\right|
$$

More generally, this kind of model requires the identification of the $R(p)$ function, and this can be achieved from a single hardening curve.

The viscoplastic case can be treated in a similar way. For instance, the viscoplastic potential can be considered as a function of the extra stress $\sigma^{\mu}$

$$
\begin{array}{llrl}
\Omega=0 & \text { if } & \bar{\sigma}-\sigma_{0}-R \leq 0 \\
\Omega=\Omega\left(\sigma^{\mu}\right) & \text { if } & \sigma^{\mu}=\bar{\sigma}-\sigma_{0}-R \geq 0
\end{array}
$$

The resulting evolution equation for $\dot{\varepsilon}^{p}$ and $\dot{p}$ are then easily obtained from (11)

$$
\dot{\varepsilon}^{p}=\left(d \Omega / d \sigma^{\mu}\right)(\partial \bar{\sigma} / \partial \sigma) \quad \dot{p}=d \Omega / d \sigma^{\mu}
$$

which is the same as (24) with $\lambda=d \Omega / d \sigma^{\mu}$. There are other possibilities, but in all cases the use of an equivalent stress allows the construction and identification of the model from uniaxial experiments.

From a physical point of view, isotropic hardening assumes a uniform augmentation of the yield stress, i.e. of the critical shear stress on each gliding system. The corresponding variable $p$ therefore reflects some scalar measure of the quantity of dislocation created by the previous plastic deformation. In particular, the extra stress $\sigma^{\mu}$ used in viscoplasticity can be interpreted as the thermally activated stress, and its scalar relation with $\dot{\varepsilon}^{p}$ is often postulated from simple physical models on dislocation motion [2].

\subsection{KINEMATIC HARDENING}

Isotropic hardening does not account for Bauschinger effect and cyclic hysteretic behaviour which are usually described through kinematic hardening, i.e. through a translation of the yield surface. This kind of model introduces a tensorial variable $X$, the back stress, which describes the position of the center of the yield surface in the stress space. The equivalent stress $\bar{\sigma}$ in the yield condition or viscoplastic potential must then be replaced by

$$
\bar{\sigma}=\bar{\sigma}(\sigma-X)
$$

For definiteness, we shall consider here a plastic model with purely kinematic hardening, but the viscoplastic and/or coupled isotropic kinematic hardening would be treated in the same way.

In the generalized standard formulation the tensorial variable $\alpha$ is introduced and the energy and dissipation inequalities are

$$
\begin{aligned}
& \psi=\psi^{e}\left(\varepsilon^{e}\right)+\frac{1}{2} \beta \alpha \cdot \alpha \\
& \sigma \dot{\varepsilon}^{p}-X \dot{\alpha} \geq 0 \quad x=\partial w / \partial \alpha=\beta \alpha
\end{aligned}
$$

The evolution equation (12) then gives from (28)

$$
\dot{\varepsilon}^{p}=\lambda \partial \bar{\sigma} / \partial \sigma \quad \dot{\alpha}=-\lambda \partial \bar{\sigma} / \partial x=\dot{\varepsilon}^{p}
$$

so that the internal variable $\alpha$ is the plastic deformation (linear kinematic hardening) and this leads to a very crude approximation of the cyclic behaviour. A better description is obtained from the so called "non linear kinematic hardening" which replaces $(30)$ by

$$
\text { (31) } \quad \dot{\varepsilon}^{p}=\lambda \partial \bar{\sigma} / \partial \sigma \quad \dot{\alpha}=\dot{\varepsilon}^{p}-\varphi \times\left|\dot{\varepsilon}^{p}\right|
$$

with a new constant $\varphi$ which together with $\beta$ can be identified from cyclic experiments. Further refinements have been proposed, introducing new internal variables, and this kind of models allows a very fine description of cyclic behaviour [8].

From a physical point of view, this back stress tensor is usually said to describe internal stresses. This interpretation will be discussed later.

\subsection{DAMAGE MODELS}

The introduction of a damage variable for the 
description of internal degradation state before rupture may also be considered as classical now. Two kinds of variables are commonly used. The first description uses some kind of porosity as damage variables with appropriate criteria and evolution equations for the different phases of damage (nucleation, growth and coalescence) [9]. These models are naturally based on plastic micromechanical analysis, and this will be discussed later.

The second kind of model is based on Kachanov's approach, introducing an effective stress tensor

(32) $\tilde{\sigma}=\sigma(1-D)^{-1}$

and characterizing the damaged behaviour by substitution of this effective stress in the undamaged constitutive equations, and in particular in the elastic energy [1]. This results in the following dissipation inequality

$$
\sigma \dot{\varepsilon}^{p}+Y \dot{D}+\ldots \geq 0
$$

with a thermodynamic force $Y$ which can be interpreted as Griffith' energy release rate. The physical meaning is less clear, but some micromechanical analysis may also be useful.

\section{THE MICROMECHANICAL TOOLKIT}

\subsection{MACROSCOPIZATION AND MICROMECHANICS}

Most of the models which are presently used in applications, and in particular for finite element analysis, are obtained from the combination of the elementary components which have been discussed above. Satisfactory results have been obtained for many problems. Much remains to be done however to fit the constitutive models and their identification to the ever increasing need for a better material description, and this requires an increased interaction between phenomenological models and microstructural characterization and understanding. This always implies some kind of "macroscopization" process, i.e. of transition from a microscopic scale towards a more macroscopic one. These processes can be viewed at different levels, and the macroworld obtained from such a process will often be used as the microworld for another scale change. For instance, the constitutive equations are written for the macroscopic volume element which is therefore the final macroscopic scale in our discussion and the ultimate macroworld in this meeting. However it may also be considered as the microworld for structure analysis and finite elements codes.

This macroscopization process can be realized in different ways, and the micromechanical analysis is one of them. It can be used whenever the microworld can be described in terms of continuum mechanics. The micro-macro transition is then a purely mechanical problem. This problem is usually a difficult one, but many methods have been developed to treat it. This kind of approach is appropriate for the description of those aspects of the macroscopic behaviour which result from microscopic heterogeneities.

A very important special case is the analysis of the polycrystalline aggregate as a population of single crystals with different orientations and shapes. The behaviour of the polycrystal and in particular its evolutive anisotropy may be described from a single crystal model and the knowledge of its initial structure. The corresponding methods from the Taylor model to the self consistent scheme have already been discussed, and we shall not further insist on them: even if some attempts have been made [11], they are usually too complicated to provide a practically useable constitutive equation.

\subsection{PERIODICAL HOMOGENIZATION}

An exact and complete derivation of the macroscopic behaviour resulting from microscopic heterogeneities may be obtained in case of a perfectly ordered microstructure, i.e. when the microscopic structure can be described from the spatial repetition of a given heterogeneous pattern. In this case, the entire microscopic structure is defined through periodicity from an elementary cell. The local stress and strain distribution inside the cell is then obtained from the resolution of a boundary value problem with periodicity conditions at the cell boundary [10]. This is already a standard procedure for the determination of elastic constants in composite materials. The application of this approach to inelastic materials can be achieved in a similar way, but it usually requires heavy computations because each loading history must be treated separately.

of course, this approach is not able to direct1y provide a constitutive equation, and it can only be used for the determination of the response of a given structure to different loading histories. This may be however very useful, in particular for the second step of the modelization, i.e. for the formulation of the constitutive equation (4). Once a micromechanism has been identified, and provided it can be simulated at a given level by a periodic material heterogeneity, it can be used to understand qualitatively and sometimes quantitatively its influence on the macroscopic behaviour and therefore on the constitutive equation. A parametric analysis may also provide some informations about the kind of parameter which can be chosen as an internal variable, i.e. as a macroscopic indicator for the investigated mechanism. It must further be noted that this micro-macro transition is thermodynamically consistent, and that the energy and dissipation potential may be transferred from the microscopic to the macroscopic level by the standard averaging process. Through the use of the standard generalized scheme, it may therefore provide a useful basis for the formulation of the evolution equation.

Besides composite materials, which are the most natural application field of these methods, many examples can be found about damage mechanisms, and some of them will be discussed later. Some examples can also be found dealing with dislocation motions and crystallographic glide at the subgrain level where the material can be considered as elastic with moving singularities and discontinuities. Some calculations have been performed for the description of cyclic hardening [12] and for the interaction of dislocation motion with obstacles [13]. Periodic homogenization therefore appears as a powerful tool for the simulation of different microscopic structures and a guideline for their modelization. 


\subsection{PLASTIC HETEROGENEITIES}

The cyclic behaviour of metais is a typical case where an important part of the observed macroscopic behaviour results from microscopic heterogeneities. The usual internal variables models, which have been presented in section 3.3 , result in a globally good description, but they result in a brutal elastic-plastic transition with discontinuous tangent modulus which is not satisfactory. This has otten been used as an argument in favour of hereditary models [4]. The microscopic underlying structure clearly is the micro-plastic heterogeneities which exist at two levels between the grains and inside each grain. The first level can be analysed through any polycrystalline model [14], but this is not enough because a similar behaviour is obtained on single crystals [15]. The second level implies the homogenization of an heterogeneous plastic material with homogeneous elasticity. The periodic homogenization described above could be used, but it would be an enormous numerical task, and its results are easily guessed: it is well known that plastic homogenization introduces an infinite spectrum of internal variables [10].

A crude but significant model can be obtained by using Voigt's homogenization technique, i.e. by assuming a uniform total strain. It is then easily shown [16] that the behaviour can be described as a Masing model, i.e. as the combination of an infinite number of springs and friction elements, Fig. 1. Such a model is entirely defined by its stiffness spectrum $g^{\prime \prime}(e)$ which can be identified from the hysteresis loop. This model allows the finite treatment of an infinite number of internal variables [17].

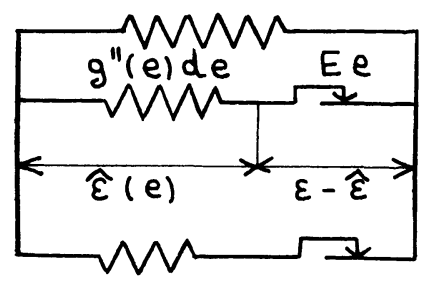

Figure 1. Masing mode

e: yield strain $\hat{\varepsilon}(e) \leq$

$g^{\prime \prime}(e)$ de: elementary stiffness

The resulting stiffness spectrum $g^{\prime \prime}(e)$ is related to the microscopic structure and heterogeneities. The evolution of this stiffness spectrum and of the hysteresis loop therefore reflects the evolution of the microstructure and for instance the development of dislocation cells. An evolutive Masing model may be used as a macroscopic model for cyclic hardening and other memory effects [17]. Within this context, the kinematic hardening variable appears as a discretization of the continuous spectrum of internal variables. In the same way, the multi-layer models which are often used [18] may be considered as higher order approximations.

\section{MICROSTRUCTURE AIDED PHENOMENOLOGY}

Many microstructural mechanisms however cannot be described in purely mechanical terms. In particu- lar, this will typically be the case when the microworld is essentially concerned with dislocation motions. The micro-macro transition cannot then be realized in quantitative terms. Microstructural characterization and understanding must then be used qualitatively and on a more intuitive basis, but, once a microstructural mechanism has been recognized as being responsible for some aspect of the behaviour, the program will remain the same with its three basic steps:

1) Choice of some macroscopic internal variable representative of the investigated mechanism;

2) Discussion of the influence of this mechanism on the behaviour:

3) Description of its own kinetics.

However, each step will remain phenomenological, and the resulting model will be identified from macroscopic experiments. In particular, the internal variables will be representative of some microscopic quantity, but their precise definition is macroscopic.

\subsection{ANELASTICITY AND STIFFNESS}

Before starting a general discussion and survey of this kind of approach, we shall begin by an illustrating example which will be treated in some detail: the influence of anelasticity on "elastic" stiffness. This investigation starts from troubles which occured when trying to define a damage variable, as suggested by (32), from the elastic stiffness reduction

$$
E(D)=E_{0}(1-D) \quad D=\left(E_{0}-E\right) / E_{0}
$$

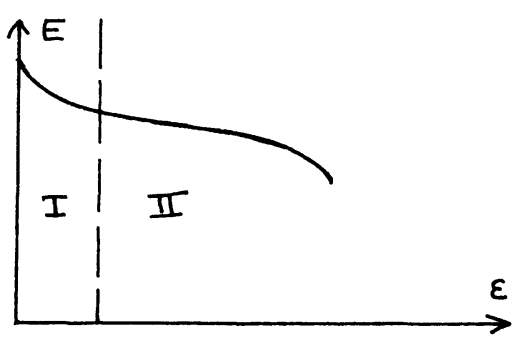

Figure 2. Stiffness evolution

The typical experimental result for the evolution of $E$ during the material lifetime is depicted in Fig.2. The regular decrease in $E$ observed in the second part of the curve may be interpreted as resulting from an increasing damage, but this is not possible for the first part where a significant decrease (up to $5 \%$ ) is observed.

From a microstructural point of view, this initial evolution of the apparent stiffness may be attributed to anelasticity, i.e. to the reversible motion of dislocations [19]. Under an applied elastic stress $\sigma$, each dislocation segment assumes a curvature $x$ proportional to $\sigma$. Denoting by $L$ the mean length of these segments between strong obstacles and by $\Lambda$ the total dislocation density, there are $\Lambda / L$ such segments per unit volume, and each of them sweeps an areax $\mathrm{L}^{3} / 12$. The contribution of this curvature to the deformation is the anelastic deformation and it can be written as 
(35) $\varepsilon^{a}=c_{1} \wedge L^{2} \sigma$

The apparent stiffness results from the superposition of this deformation with the elastic part $\varepsilon^{e}=\sigma / E_{0}$

$$
E=\left(1 / E_{0}+c_{1} \Lambda L^{2}\right)^{-1} \approx E_{0}\left(1-c_{1} E_{0} \Lambda L^{2}\right)
$$

which shows that anelasticity decreases the observed stiffness. In order to evaluate the evolution of this anelastic correction, it must be noted that $L$ is the mean distance between strong obstacles, and that $1 / L^{2}$ is their average number per unit surface in the gliding plane. These obstacles come from other dislocations with a density proportional to $\Lambda$ and from other obstacles such as impurities. The stiffness (36) can then be written as

$$
E=E_{0}\left[1-c_{2} \Lambda /\left(\Lambda+\Lambda_{1}\right)\right]
$$

with $\Lambda_{1}$ being some measure of the impurity -induced obstacle density. This explains the observed decrease in $E$ : starting from an initial state with low $\Lambda, E$ decreases with increasing plastic strain to the limit value $E_{0}\left(1-c_{2}\right)$. An evaluation of $c_{2}$ may show that this value is consistent with the observed stiffness reduction [19].

Starting from this microstructural analysis, a phenomenological model can be proposed for the description of this initial stiffness reduction: as discussed in section 3.2 , the variable $p$ is the macroscopic indicator for the dislocation density. It is then reasonable from (37) to assume $E=E(p)$

$$
\psi^{e}=\psi^{e}\left(\varepsilon^{e}, p\right)=\frac{1}{2} E(p) \varepsilon^{e 2}
$$

and for instance

$$
E(p)=E_{0}\left[1-c_{0} p /\left(p+p_{0}\right)\right]
$$

with two new constants $p_{0}$ and $c_{0}$ to be identified from experiments.

\subsection{DISLOCATION MOBILITY}

The mobility of dislocations is an important microstructural concept which is usually absent from phenomenological models. A significant improvement would be to include it in the macroscopic models. The concept of isotropic hardening for instance conveys the fact that, roughly speaking - but phenomenologists always speak roughlythe yield limit, i.e. the critical shear stress on every gliding systems, is an increasing function of the dislocation density which is represented by the macroscopic variable $p$ which, due to the plasticity formalism, is identified to some equivalent plastic strain. The justification for this is the fact that plastic deformations always occur through the creation of dislocation loops, and therefore increase the dislocation density.

According to Orowan's formula however, the plastic deformation resulting from the motion of a dislocation line is proportional to the swept area. Once a dislocation line is created, the amount of plastic deformation which can be generated is therefore proportional to the distance it will be allowed to travel before being stopped by obstacles: the mean free path. From a phenome- nological point of view, this can be taken into account by the introduction of a new internal variable 1 to represent this mean free path. The influence of 1 will be essentially felt in the relation between the increments of dislocation density $p$ and equivalent strain $\bar{\varepsilon}$, for instance

(40) $\mathrm{d} \bar{\varepsilon}=1 \mathrm{dp}$

if 1 is normalized in such a way that it is 1 in the reference state $(d \bar{\varepsilon}=d p)$

This kind of model will be useful when the mean free path is subject to significant change for different loading histories. The creation and development of dislocation structures in particular may introduce significant changes in 1 . A model has been developed along this line for the description of path-dependent hardening in hotrolled steel sheet [2]. This model was formulated as in section 3.2 with (25) replaced by (40) with an appropriate path dependent evolution law for 1. Another case where this kind of model can be used is the description of plastic deformation at changing temperature (prevision of the residual stresses after quenching for instance): a model with isotropic hardening may be identified at each temperature and the evolution of $p$ under changing $\varepsilon$ and $\theta$ can be described by $(40)$ with an appropriate temperature dependent $1(\theta)$.

\subsection{TEMPERATURE INDUCED TRANSFORMATION}

More generally, the formulation of a temperature dependent constitutive equation which would be able to describe the behaviour under varying temperature is a difficult task. The first step always is the formulation of an isothermal mode 1 at each temperature. This is usually achieved by using a simple model with temperature dependent material constants. In case of time dependent behaviour, this is usually completed by some timetemperature equivalence principle. This first step results in a model parametrized by the temperature, which is a reasonable assumption in the absence of microstructural transformations. The numerical implementation of this model together with the thermomechanical coupling may be not so easy, but this is not our interest here.

The situation however is quite different and much more complicated when temperature changes induce microstructural transformations. The case of the martensitic transformation is probably the most important, and we shall concentrate our attention on it. Once the transformation has been identified, a proper choice of internal variables must be made. In most cases, they will be taken as the concentrations of one or several constituents according to the number of concerned phases: the simplest model of martensitic transformation considers only the transformation austenite $\gamma \rightarrow$ martensite $\alpha^{\prime}$ and the concentration $\alpha$ of martensite is a good internal variable, but more sophisticated model may be considered taking into account for instance the transformation austenite $\gamma \rightarrow$ Martensite $\varepsilon \rightarrow$ Martensite $\alpha^{\prime}$ which will require two concentrations or also several martensitic variants.

Constitutive equations must then be postulated. This will usually be done through some homogenization processes which may range from the simplest melange law resulting from Voigt's approximation to a sophisticated self consistent scheme. 
An evolution equation must then be postulated describing the kinetics of the phase transformation. This equation must be non linear, in order to take into account a temperature dependent equilibrium concentration [20]. A typical form may be

(41) $d \alpha=(1-\alpha) f(\alpha) d t, f=f_{0}\left[(1-\alpha) \alpha_{0}-\left(1-\alpha_{0}\right) \alpha\right]$

where the transformation velocity $f(\alpha)$ vanishes for $\alpha=\alpha_{0}$, the equilibrium concentration. The thermodynamic framework of generalized standard materials may also be used [21].

More generally, the description of microstructural phase transformation and its coupling with plastic deformation (transformation plasticity, ageing,...) is a difficult problem. Further development will require a deep microstructural understanding.

\section{COMBINED APPROACHES}

For many applications, the methodological tools which have been described above will have to be combined. This will be illustrated in some domains which have been widely investigated, and where the interaction between mechanics and microstructure is already operative.

\subsection{THE PHENOMENOLOGY OF SINGLE CRYSTAL}

As discussed earlier, many microscopic levels can be considered, and the single crystal is one of the most important. In particular, it is the appropriate microworld for any model of polycrystalline aggregate which must therefore include a single crystal model as one of its material inputs. Since it can be described in terms of continuum mechanics, it may be considered as the basic macroworld in such a meeting between physicists and mechanicians, and among the questions to be discussed here, in Aussois, the formulation of a reliable constitutive equation for the single crystal is the most obvious one.

In this formulation, many features are imposed by the gliding mechanism and its crystallographic nature. It is generally accepted that the motion of a single crystal results from the elastic (lattice) deformation $\mathrm{d} \varepsilon^{\mathrm{e}}$, the crystallographic lattice rotation $d \omega^{l}$ and the plastic distorsion which results from the superposition of elementary shears $d \gamma^{s}$ on each gliding system $s\left(n^{s}, g^{s}\right)$

$$
\begin{aligned}
& d u_{i, j}=d \varepsilon^{e}{ }_{i j}+d \omega_{i j}+d \beta^{p_{i j}} \\
& d \beta^{p_{i j}}=\Sigma d \gamma^{s} g_{i} n^{s}{ }_{j}
\end{aligned}
$$

where small perturbations have been assumed, but large plastic deformation and lattice rotations can be treated without significant change [7]. It is also generally accepted that in most cases the slip rate $\dot{\gamma}^{s}$ on each system essentially depends on the corresponding resolved shear stress

$$
\tau^{s}=g^{s} \sigma n^{s}=\sigma_{i j} g^{s}{ }_{i} n_{j}
$$

but different reasons may be given for this: from a physical point of view, this is some kind of generalized Schmid's law, while from a mechanical point of view, this is justified by showing $\tau^{s}$ to be the thermodynamical force associated to $\dot{\gamma}^{s}$

(45) $\quad \sigma_{i j} \dot{\varepsilon}^{p}{ }_{i j}=\Sigma \tau^{s} \dot{\gamma}^{s}$
The basis physical content of a single crystal model is therefore included in this slip law and its evolution. It is usually postulated in phenomenological terms, and the general structures which have been discussed above for a $(\sigma, \varepsilon)$ constitutive equation can be translated to this $\left(\tau^{s}, \dot{\gamma}^{s}\right)$ relationships which at a fixed stage can be written as (21) with different special cases, as described in Figure 3. Examples of each of these cases can be found in the literature

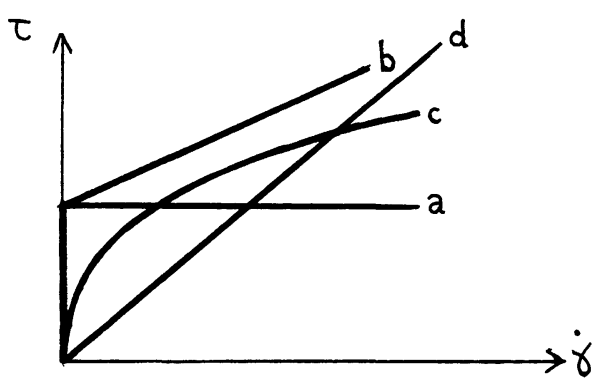

Figure 3. Plastic and viscoplastic slip law a rate independent plasticity b Bingham viscoplasticity c Norton Hoff viscoplasticity d linear viscous law

The next step is to follow the intragranular hardening, i.e. the evolution of this slip law, and in particular of the critical shear stress $\tau^{\mathrm{s}}{ }_{c}$ in case $a$ or $b$. This is usually expressed in terms of an hardening matrix which relates the increment of $\tau^{s}$ to the elementary slips $d y^{s}$. This is essentially some generalization of isotropic hardening as described in section 3.2 . Some attempts however have been made to use more sophisticated hardening laws [22] including for instance linear or non linear kinematic hardening. Similarly, the discussion of section 5.2 can also be translated in terms of the slip law by introducing the dislocation densities $a^{s}$ instead of $\gamma^{s}[7]$.

More generally, there is a close mechanical and thermodynamical analogy between the plastic constitutive equation and hardening and the slip law on a gliding system. This analogy is quite helpful and any model or result obtained at one level can be transferred to the other. It can even be made more complete by remarking that anelasticity, as described in section 5.1, may be considered as an elastic slip.

\subsection{HOMOGENIZATION APPROACH TO DAMAGE}

As discussed in section 3.4 , the mechanical concept of damage is always associated to the macroscopic averaging of a population of microdefects (microcracks or microcavities). At a microscopic level, the concentration, distribution, localization, orientation and shape of these defects can be analysed and described. Since these defects essentially introduce a material discontinuity, their influence may be analysed in micromechanical terms. Periodical homogenization in particular is usually quite appropriate. 
A typical procedure [23] may be the following. Starting from a microscopic observation of the microdefects, a representative cell is constructed and parametrized by one variable. For instance, assuming some kind of cavitation, the microscopic damage will be simulated by a regular array of spherical holes. The damage internal variable may then be defined as the ratio $a=r / 1$ of the hole radius $r$ to the fixed cell dimension 1. The homogenization procedure can then be activated, resulting in a damaged material response. For instance, the damaged elastic behaviour is characterized by the elastic stiffness $c(a)$ leading to

(46) $\psi^{e}=\frac{1}{2} c(a) \varepsilon^{e} \varepsilon^{e}$

where $c(a)$, i.e. the Lamé constants $\lambda(a), \mu(a)$, results from the homogenization computation as a known decreasing function of $a$. The thermodynamic force $Y$ associated to $a$ is

$$
Y=-\frac{1}{2} c^{\prime}(a) \varepsilon^{e} \varepsilon^{e}
$$

and a fatigue evolution law may be postulated as for instance

$$
d a=\left(Y / Y_{0}\right)^{n} d N
$$

Different kinds of defect configuration have been analysed in this way. Microcavitation of course, but also microcracks with the description of the pseudo plastic behaviour which results from dry friction on the crack [24], interface degradation in composite materials [25], etc... Micromechanics, and periodic homogenization in particular, thus allows a characterization of the damaged behaviour resulting from some identified microscopic damage mechanism.

\subsection{INTERNAL STRESSES}

The concept of internal stress is often mentioned as a significant microstructural variable, and it is often introduced in phenomenological models (kinematic hardening for instance). This concept is essentially associated to any micro-macro transition: since the macrostress is the averaged microstresses, the latter do not vanish after a macro unloading. The residual values are the internal stresses, and they define a self equilibrated stress field with zero mean value. Different levels of internal stresses are usually considered, and they are associated to the different micro-macro transitions: Type I internal stresses are related to structure analysis which has been interpreted in section 4.1 as a micro-macro transition; Type II refers to the micromechanical transition from a locally heterogeneous microcontinuum to an homogenized macrovolume element; and Type III refers to the physical transition from the crystal with its defects to the locally homogeneous single crystal.

At each level, the local stress may therefore be considered as the sum

(49) $\sigma=\sigma^{\text {ext }}+\left\langle\sigma^{i}\right\rangle+\tilde{\sigma}^{i}$

where oxt is the external applied stress, while the internal stress $\sigma^{j}$ has been decomposed into its mean value $\left\langle\sigma^{i}\right\rangle$ and a fluctuating part $\tilde{\sigma}^{i}$. Local plastic deformations will occur when the local stress reaches a critical value $\sigma_{c}$, but a macro plastic strain requires this condition to be satisfied everywhere

$$
\left|\sigma^{\text {ext }}+\left\langle\sigma^{i}\right\rangle\right| \geq \sigma_{c}+\sup \left|\tilde{\sigma}^{i}\right|
$$

This is the justification for the usual argumentation that kinematic hardening is related to the mean value of the internal stress, while isotropic hardening results from their fluctuations. Unfortunately, this is not so easy, because the internal stresses must be self equilibrated with zero mean value, so that at each level $\left\langle\sigma^{1}\right\rangle=0$; there can be no kinematic hardening in an homogeneous material.

In case of a two-phases medium, on the contrary, things are perfectly clear: the mean value over the whole body vanishes but this only means that

(51) $f\left\langle\sigma^{i}{ }_{1}\right\rangle+(1-f)\left\langle\sigma^{i}\right\rangle=0$

where $\sigma^{i}$ and $\sigma^{i}{ }_{2}$ denote the internal stresses in the two phases and where $f$ is the volume fraction of phase 2. Plasticity will occur when condition (50) is satisfied in the weakest phase directly leading to a kinematic hardening. More precisely, it can easily be shown that the Voigt homogenization (section 4.3) gives exactly, in this case, the usual Prager model.

This is known to be the fundamental hardening mechanism in case of hard inclusions in a soft matrix [26] and this approach has been used to relate the cyclic behaviour in metals to the development of dislocation structures (soft cells with hard walls) [27]. More generally cyclic plastic behaviour results from plastic heterogeneities and kinematic hardening may be considered as a first approximation of the complete behaviour as discussed in section 4.3. The back stress can then in some sense be interpreted as a mean internal stress in the weak part of the material. But this is a qualitative interpretation in the line discussed in section 5 more than a quantitative one and it will be quite difficult to relate it to the internal stress which can be measured using for instance $X$-ray diffraction techniques [28].

From an experimental point of view, this internal stress is often "measured" in a dip test

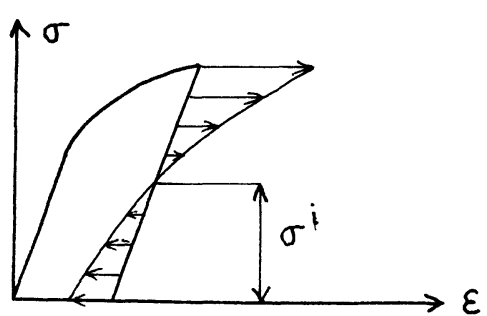

Figure 4. Dip test experiment.

experiment, which consists in partial unloading followed by creep. The internal stress is obtained when the initial creep rate vanishes (Fig.4). In the case of a viscoplastic model with kinematic hardening and without an elastic domain, this procedure indeed gives an experimental value of $X$, but this interpretation is strongly model de- 
pendent and Polak \& Klesnil have shown [16] that this $\sigma^{i}$ can be given an entirely different signification for a viscoplastic Masing model generalizing the plastic model of section 4.3. This experiment therefore allows an experimental identification of $X$ for a given model, but it is not sure that this internal stress has a precise physical meaning and can be related to the microscopic internal stress.

Although it is frequently mentioned, the relation between kinematic hardening and internal stresses is therefore not so clear.

\section{CONCLUSION}

Phenomenological models for the description of metal plasticity can be developed in many different ways and, although their general structure is always the same, they may take quite different forms. Their final aim is to combine relative simplicity, a great versatility, and a good description of reality, and, to achieve this, all means are good. Much has been achieved by phenomenology alone, and this will go on in the future, but there has been an important trend in recent years to include more physics in these models, and we have presented here the basis on which this can be done and the kind of information which is needed. The door is open, and a wide field lies before us.

Acknowledgements. Most of the ideas which have been presented here results from many discussions with many friends in Aussois and elsewhere inside the GRECO Grandes Déformations et Endommagement. Let them all be thanked.

\section{REFERENCES}

[1] J.LEMAITRE \& J.L.CHABOCHE, Mécanique des Matériaux Solides, Paris, Dunod, 1985.

[2] F.SIDOROFF \& C.TEODOSIU, Microstructure and phenomenological models for metals, Large Deformations of polycrystalline aggregate, Ed. J.Gittus, Paris, 1985.

[3] P.germain, Cours de Mécanique des Milieux Continus, Paris, Masson, 1973.

[4] P.GUELIN, Remarques sur l'hystérésis mécanique, J. Mécan., vol.19, 1980, p.217.

[5] K.C.VALANIS, A theory of viscoplasticity without a yield surface, Arch. Mech. vol.25, 1971, p.517.

[6] J.J.MOREAU, Sur les lois de frottement, plasticité, viscosité, C.R.A.S. Paris, Série A, tome 271,1970, p.608.

[7] C.TEODOSIU \& F.SIDOROFF, A theory of finite elasto-viscoplasticity of single crystals, Int. J. Engng. Sc., vol.14, 1976, p.165.

[8] A.BENALLAL \& D.MARQUIS, Constitutive equations describing non proportional cyclic plasticity effects, J. Engng. Mat. and Techn., to be published.

[9] F.MUDRY, A.PINEAU, J.C.DEVAUX \& G.ROUSSELIER, Numerical and experimental validation of a local ductile rupture criterion in terms of critical void growth, 3rd Intern. Symp. on non linear fracture Mechanics, Knoxville, 1986.

[10] P.SUQUET, Plasticity of highly heterogeneous media, XVI ICTAM, Lyngby, 1984

[11] M.ARMINJON, Théorie d'une classe de modèles de Taylor hétérogènes, Application aux textures de déformation des aciers, Acta Met., vol.35, 1987, p. 615 .

[12] F.KRASUCKI, Modélisation de la fatigue par un procédé d'homogénéisation, J. Mécan. Théor. et App1., vol.5, 1986, p.629.
[13] M.RACHADI, Etude par homogénéisation des comportements microstructuraux avec glissement, Thèse, Paris, 1986.

[14] J.H.DRIVER \& P.RIEUX, The cyclic stressstrain behaviour of polycrystalline $A 1-5 \% \mathrm{Mg}$, Mat. Sc. Engng, vol.68, 1984, p. 35 .

[15] H.MUGHRABI, The cyclic hardening and saturation behaviour of copper single crystals, Mat. Sc. Engng., vol.33, 1978, p.207.

[16] J.POLAK \& M.KLESNIL, The hysteresis loop, Fat. Eng. Mat. Struc., vol.5, 1982, p.19.

[17] F.SIDOROFF, Evolutive Masing model, cyclic plasticity, ageing and memory effects, SMIRT 9, Lausanne, 1987.

[18] P.DELOBELLE, Sur les lois de comportement viscoplastique à variables internes, ou, peut-on modéliser simplement des phénomènes complexes, J. Phys., to be published.

[19] A.HAMEL, A.VINCENT, J.CHICOIS, C.MAI \& R.FOUGERES, on the fatigue behaviour of pure polycrystalline aluminium studied by unloading stiffness evolution, Fatigue 84 , Birmingham, 1984 .

[20] J.B.LEBLOND \& J.DEVAUX, A new kinetic mode1 for anisothermal metallurgical transformations in steel, Acta. Met., vol.32, 1984, p.137.

[21] J.GIUSTI \& R.RADENKOVIC, A model of thermo mechanical behaviour of metals with phase changes, Mech. Res. Comm., vol.10, 1983, p.163.

[22] J.H.SCHMITT, Contribution à l'étude de 1 a micro-macroplasticité des aciers, Thèse, Grenoble, 1986.

[23] J.J.MARIGO, Modelling of brittle and fatigue damage for elastic material by growth of microvoids, Plasticity today, Udine, 1983. 
[24] E.SANCHEZ-PALENCIA \& D.LEGUILLON, On the behaviour of a cracked elastic body with or without friction, J. Mech. Th. et Appl., vol.1, 1982, p.195.

[25] F.LENE, Damage constitutive relations for composite materials, Engng. Fract. Mech., to be published.

[26] M.F.ASHBY, The deformation of plastically non-homogeneous materials, vol.21, 1970, p.399.

Phil.Mag.

[27] H.MUGHRABI, T.UNGAR, W.KIENLE \& M.WILKENS Long range internal stresses and asymmetric $x$-ray line broadening in tensile deformed <001>-oriented copper single crystals, Phil.Mag. A, 1986, vol. $53 n^{\circ} 6$, p. 793.

[28] G.MAEDER, X-ray diffraction and stress measurement, Chemica Scripta, 1986, vol.26 A, p.23. 